EPJ Web of Conferences 81, 02022 (2014)

DOI: 10.1051 /epjconf/ 20148102022

(C) Owned by the authors, published by EDP Sciences, 2014

\title{
Investigation of the structure of the few body kaonic nuclei using the method of hyperspherical functions in momentum space
}

\author{
Roman Ya. Kezerashvili ${ }^{1, a}$ and Sh. M. Tsiklauri ${ }^{2}$ \\ ${ }^{1}$ New York City College of Technology, The City University of New York, USA \\ ${ }^{2}$ Borough of Manhattan Community College, The City University of New York, USA
}

\begin{abstract}
The kaonic clusters $\bar{K} N N$ and $\bar{K} N N N$ are studied within the method of hyperspherical functions in the momentum representation. Binding energies and widths of three- and four-body nuclear quasibound states are calculated by employing realistic $N N$ potentials and the energy dependent chiral $\bar{K} N$ interaction, as well as a phenomenological $\bar{K} N$ potential. The comparison of the results of calculations obtained in the framework of the variational method, Faddeev, Faddeev-Yakubovsky equations, and method of hyperspherical functions in configuration space are discussed.
\end{abstract}

\section{Introduction}

The study of antikaon interactions with nucleon and nuclei has a long history and during the last decade attract many attentions after Akaishi and Yamazaki [1] predicted the existence of deep and narrow $\bar{K}$ bound states in $\bar{K}$-nuclear few body clusters by calculating their binding energy within the framework of the Brueckner-Hartree-Fock theory. Due to the strong $\bar{K} p$ interaction $\bar{K}$ mesons are expected to form light kaonic clusters $\bar{K} N N, \bar{K} N N N$, and $\bar{K} N N N N$ beginning with the $\bar{K} p p$. The report summarizes our understanding of $\bar{K}$-nuclear interactions and reviews the present theoretical situation in the quest for quasibound antikaon-nuclear systems is presented in Ref. [2]. The light kaonic clusters $\bar{K} N N$ and $\bar{K} N N N$ represent a three- and four-body systems and theoretically can be treated in the framework of a few-body physics approaches: the variational method [1], [3] - [6], the method of Faddeev [7] - [13] and Faddeev-Yakubovsky [14] equations and the method of hyperspherical harmonics (HH) [15]. Variational calculations have been focused on using a phenomenological $\bar{K} N$ potential or $\bar{K} N$ effective interactions based on chiral SU(3) dynamics [16], [4], [5], while three-body calculations solving Faddeev equations in the formulation of Alt-Grassberger-Sandhas were used separable interactions. Binding energies and widths of three-body $\bar{K} N N$ and four-body $\bar{K} N N N$ bound states were calculated in the hyperspherical basis using realistic NN potentials and the energy dependent chiral $\bar{K} N$ interactions. For the theoretical calculations for the simplest antikaon-nuclear system, the $\bar{K} N N$ cluster with total isospin $I=\frac{1}{2}$ for studying the role of the antikaon as a possible mediator to bind two baryons which would otherwise not form a bound state, one encounters a broad band of binding energies ranging between about 16 and $80 \mathrm{MeV}$, while the decay widths cover values between 40 and $110 \mathrm{MeV}$. The Faddeev approaches lead to binding energies higher than the variational approach.

\footnotetext{
a e-mail: rkezerashvili@ citytech.cuny.edu
} 
The comparison of results of calculations for bound states of the kaonic cluster $\bar{K} N N N$ obtained by using separable potential models for the $N N$ and $\bar{K} N$ interactions and by employing the realistic $N N$ potential and the energy dependent chiral $\bar{K} N$ interaction shows deep binding of $\bar{K} N N N$ in contrast to the weak binding result based on the chiral SU(3) dynamics model of the $\bar{K} N$ interaction.

In this report we present three- and four-body nonrelativistic calculations within the framework of the potential model for the $\bar{K}$-nuclear clusters $\bar{K} N N$ and $\bar{K} N N N$ using the hyperspherical basis in momentum representation. In our calculations we are using two type of realistic $N N$ interactions and $\bar{K} N$ interactions derived within a chiral model [16], as well as the energy independent $\bar{K} N$ interaction given in Ref. [1]. This provides the understanding of the dependence of the binding energy on the $N N$ interaction and an importance of the $\bar{K} N$ interaction in the formation of the light kaonic clusters $\bar{K} N N$ and $\bar{K} N N N$.

\section{Theoretical framework}

The hyperspherical harmonics $(\mathrm{HH})$ method represents a technique to describe the bound and scattering states for a few body system. The main idea of this approach is the expansion of the wave function of the corresponding nuclear states in terms of hyperspherical harmonics that are the eigen functions of the angular part of the Laplace operator in the six-dimensional space (three-body problem) or in the nine-dimensional space (four-body problem). The review of the research performed within this method can be found in the monographs [17] - [18]. In our calculations we use the HH method in momentum representation [19]. In this approach after the introduction of the trees of Jacobi coordinates for three- or four-particle system $\mathbf{x}_{i}=\frac{1}{\sqrt{l(l+1)}}\left[\sum_{j=1}^{l} m_{j} \mathbf{r}_{j}-l \mathbf{r}_{j+1}\right], l=1,2,3, \ldots N$, where $m_{j}$ and $\mathbf{r}_{j}$ are the particles masses and position vectors, respectively and $N$ is the number of particles, follow Refs. [19], [18] one starts from the integral form of the Schrodinger equation for the threeor four-particles, respectively, and then rewriting this equation in momentum representation using the set of the Jacobi momenta $\mathbf{q}_{i}$ in 3(N-l)-dimensional momentum space conjugated to the coordinate presented above. After that one introduces the set of the hyperspherical coordinates in the momentum space given by the hyperradius $\varkappa^{2}=\sum_{l=1}^{N} q_{l}^{2}$ and the set of angles $\Omega_{\varkappa}$, which define the direction of the vector $\varkappa$ in $3(N-l)$-dimensional momentum space, as well as the symmetrized hyperspherical harmonics in momentum representation $\Phi_{\mu}^{\lambda}\left(\Omega_{\varkappa}, \sigma, \tau\right)$ that are written as a sum of products of spin and isospin functions and hyperspherical harmonics. Above, for the sake of simplicity, we denoted by $\lambda$ the totality of quantum numbers on which the $N$-body hyperspherical harmonics depend and the integer $\mu$ is the global momentum in the $3(N-l)$-dimensional configuration space, which is the analog of angular momentum in case of $N=2$. The $\mathrm{HH}$ are the eigenfunctions of the angular part of the 3(N-l)-dimensional Laplace operator in configuration space with eigenvalue $L_{N}\left(L_{N}+1\right)$, where $L_{N}=\mu+3(N-2) / 2$ and they are expressible in terms of spherical harmonics and Jacobi polynomials [17], [18]. By expanding the wave function of $N$ bound particles in terms of the symmetrized hyperspherical harmonics in momentum space

$$
\Psi\left(\varkappa, \Omega_{\varkappa}\right)=\varkappa^{-\frac{3 N-4}{2}} \sum_{\mu, \lambda} u_{\mu}^{\lambda}(\varkappa) \Phi_{\mu}^{\lambda}\left(\Omega_{\varkappa}, \vec{\sigma}, \vec{\tau}\right),
$$

and substituting Eq. (1) into the integral Schrodinger equation in the momentum representation one obtains a system of coupled integral equations for the hyperradial functions $u_{\mu}^{\lambda}(\varkappa)$. 

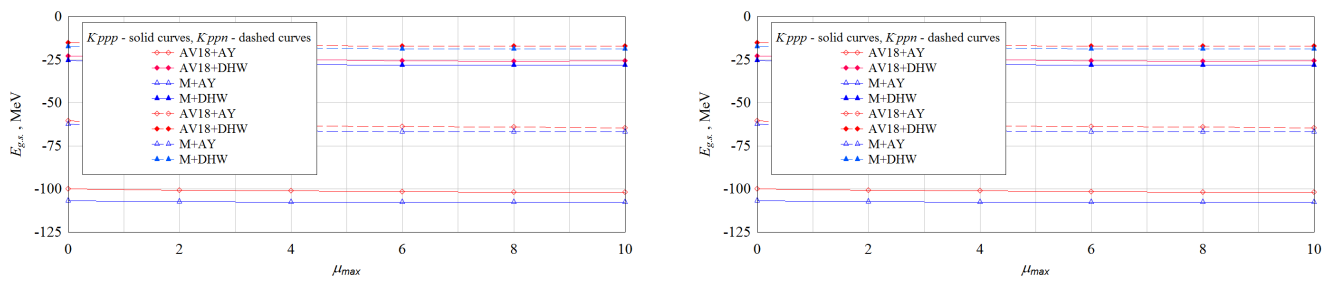

Figure 1. The convergence of the ground state energies of the kaonic clusters $\bar{K} p p, \bar{K} p p p$ and $\bar{K} p p n$.

\section{Results and discussion}

In the calculations for the $N N$ interaction we used the Argonne AV18 potential and Minnesota (M) potential [20], while for $\bar{K} N$ interaction we use the energy dependent effective interaction derived based on chiral SU(3) meson-baryon dynamics $\bar{K} N$ interaction [4], [5] that we refer as DHW potential with the range parameter $b=0.47 \mathrm{fm}$ and a phenomenological $\bar{K} N$ potential [1] that we refer as AY potential with the range parameter $b=0.66 \mathrm{fm}$. The symmetrized hyperspherical harmonics $\Phi_{\mu}^{\lambda}\left(\Omega_{\varkappa}, \sigma, \tau\right)$ for the system $\bar{K} p p$ were antisymmetric with respect to the permutation of protons and the symmetrized $\Phi_{\mu}^{\lambda}\left(\Omega_{\varkappa}, \sigma, \tau\right)$ for the system $\bar{K} N N N$ were built based on the three-body Raynal-Revai coefficients [21], [22]. To find the binding energies with above mentioned set of potentials we solve a system of coupled integral equations for the hyperradial functions $u_{\mu}^{\lambda}(\varkappa)$ with $\mu_{\max }=10$.

The convergence of ground state energy are shown in Fig. 1 as a function of $\mu_{\max }$. Using the wave function obtained for $\mu_{\max }=10$ the width was evaluated through the expression $\Gamma=-2\left\langle\Psi\left|\operatorname{Im} V_{\bar{K} N}\right| \Psi\right\rangle$, where $V_{\bar{K} N}$ sums over all pairwise $\bar{K} N$ interactions. In Table 1 we present our results for $\bar{K} p p$ that we compare with those obtained by the different methods. Results of our calculations for the energy and the width show dependence on the $N N$ potentials and on the $\bar{K} N$ interactions. However, this dependence is dramatically different: for the same $\bar{K} N$ interaction and different $N N$ potentials the ground state energy and the width are changed by about $20 \%$, while for the same $N N$ potential and different $\bar{K} N$ interaction the energy is changed by the factor more than 2.5 and the width is changed by more than twice. The same tendency can be observed from Table 2 that presents the results of calculations for the ground state energy and width for $\bar{K} p p n$ and $\bar{K} p p p$.

Table 1. Ground state energy and width for $\bar{K} p p$.

\begin{tabular}{|c|c|c|c|c|c|c|c|c|}
\hline & \multicolumn{6}{|c|}{ Present work } & & \\
\hline & $\mathrm{AV} 18+\mathrm{AY}$ & AV18 & + DHW & $\mathrm{M}+\mathrm{AI}$ & $\mathrm{N}$ & I+DHW & & \\
\hline$B, \mathrm{MeV}$ & 39.8 & & 4.9 & 48.3 & & 16.9 & & \\
\hline \multirow[t]{3}{*}{$\Gamma, \mathrm{MeV}$} & 74.6 & & 6.5 & 95.8 & & 43.2 & & \\
\hline & \multicolumn{4}{|c|}{ Faddeev Equations } & \multicolumn{3}{|c|}{ Variational Method } & $\mathrm{HH}$ \\
\hline & [7]-[8] & 9]-[11] & [13] & [14] & [1] & {$[4]-[5]$} & [6] & [15] \\
\hline$B, \mathrm{MeV}$ & $50-70$ & $16-80$ & 35 & 51.5 & 48 & $20 \pm 3$ & $40-80$ & 15.7 \\
\hline$\Gamma, \mathrm{MeV}$ & $90-100$ & $47-75$ & $50-80$ & & 61 & $40-70$ & $40-85$ & 41.2 \\
\hline
\end{tabular}


Table 2. Ground state energy and width for $\bar{K} N N N$.

\begin{tabular}{c|c|cccc}
\hline \multicolumn{2}{l|}{} & AV18+AY & AV18+DHW & M+AY & M+DHW \\
\hline $\bar{K} p p n$ & $B, \mathrm{MeV}$ & 64.6 & 17.2 & 66.7 & 18.7 \\
& $\Gamma, \mathrm{MeV}$ & 74.2 & 27.1 & 80.4 & 31.4 \\
\hline $\bar{K} p p p$ & $B, \mathrm{MeV}$ & 101.9 & 25.8 & 107.6 & 28.1 \\
& $\Gamma, \mathrm{MeV}$ & 19.8 & 28.1 & 19.7 & 31.2
\end{tabular}

For the comparison let's mention that in Ref. [15] for the binding energy and the width of $\bar{K} p p n$ cluster obtained $18.5 \mathrm{MeV}$ and $31.0 \mathrm{MeV}$ and for $\bar{K} p p p 29.3 \mathrm{MeV}$ and $32.6 \mathrm{MeV}$, while calculation within the Faddeev-Yakubovsky equations gives $69 \mathrm{MeV}$. The comparison our results for $\bar{K} p p, \bar{K} p p n$ and $\bar{K} p p p$ obtained for AV18 NN interaction and DHW $\bar{K} N$ interaction with calculations in Ref. [15] within the variational HH method for AV14 NN interaction and DHW $\bar{K} N$ interaction shows that they are very close.

Based on the results of our calculations we can conclude that the pairwise $\bar{K} N$ interaction plays the major role in the formation of the kaonic bound state and we found that $\bar{K} N$ effective interactions [4], [5] based on chiral SU(3) dynamics [16] gives relatively modest binding for the $\bar{K} p p, \bar{K} p p n$ and $\bar{K} p p p$.

\section{References}

[1] Y. Akaishi, T. Yamazaki, Phys. Rev. C 65, 044005 (2002); T. Yamazaki, Y. Akaishi, Phys. Lett. B 535 (2002) 70.

[2] W. Weise, Nucl. Phys. A 835, 51-58 (2010).

[3] A. Doté, W. Weise, Prog. Theor. Phys. Suppl.168, 593-597 (2007).

[4] A. Doté, T. Hyodo, W. Weise, Nucl. Phys. A 804, 197-206 (2008).

[5] A. Doté, T. Hyodo and W. Weise, Phys. Rev. C 79, 014003 (2009).

[6] S. Wycech, A. M. Green, Phys. Rev. C 79, 014001 (2009).

[7] N.V. Shevchenko, A. Gal, J. Mares, Phys. Rev. Lett. 98, 082301 (2007).

[8] N.V. Shevchenko, A. Gal, J. Mares, J. Révai, Phys. Rev. C76, 044004 (2007).

[9] Y. Ikeda, T. Sato, Phys. Rev. C76, 035203 (2007).

[10] Y. Ikeda, T. Sato, Phys. Rev. C79, 035201 (2009).

[11] Y. Ikeda, H. Kamano, T. Sato, Prog. Theor. Phys. 124, 533 (2010).

[12] E. Oset, D. Jido, T. Sekihara, A. Martinez Torres, K.P. Khemchandani, M. Bayar, J. YamagataSekihara, Nucl. Phys. A 881, 127-140 (2012).

[13] M. Bayar, E. Oset, Nucl. Phys. A 883, 57(2012); Nucl. Phys. A 914, 349-353 (2013).

[14] S. Maeda, Y. Akaishi, T. Yamazaki, Proc. Jpn. Acad., Ser. B 89, 418-437 (2013).

[15] N. Barnea, A. Gal, E. Z. Liverts, Phys. Lett. B 712, 132-137 (2012).

[16] T. Hyodo and W. Weise, Phys. Rev. C 77, 035204 (2008).

[17] J. Avery, Hyperspherical Harmonics, Kluwer Academic, Dordrecht, 1989.

[18] R.I. Jibuti, K.V. Shitikova, Method of hyperspherical functions in atomic and nuclear physics. Energoatomizdat, Moscow, 270p. 1993. (in Russian).

[19] R.I Jibuti, N.B. Krupennikova, V. Yu. Tomchinsky, Nucl. Phys. A 276, 421-435 (1977).

[20] D.R. Thompson, M. LeMere, Y.C. Tang, Nucl. Phys. A286, 53, ( 1977).

[21] R.I. Dzibuti, Sh. M. Tsiklauri, Yad. Fiz. 36, 1387 (1982); R.I. Dzibuti, N.B. Krupennikova, L.L. Sapkisian, Sh. M. Tsiklauri,Yad. Fiz. 44, 349 (1986).

[22] R.I. Jibuti, R.Ya. Kezerashvili, Nucl. Phys. A 437, 687 (1985). 